\title{
Prevalence of Toxocariasis and Its Risk Factors in Patients with Eosinophilia in Korea
}

\author{
Hyun Beom Song ${ }^{1, \dagger}$, Deokho Lee ${ }^{1, \dagger}$, Yan Jin², Jinwoo Kang,", Shin-Hyeong Cho ${ }^{4}$, Min Sun Park ${ }^{5}$, Jin-Ho Park ${ }^{5,6}$, \\ Woo-Jung Song ${ }^{7, \#, *}$, Hye-Ryun Kang ${ }^{7}$, Sang Hyub Lee ${ }^{3}$, Sung-Tae Hong ${ }^{1}$, Min-Ho Choi',** (i) \\ ${ }^{1}$ Department of Tropical Medicine and Parasitology and Institute of Endemic Diseases, Seoul National University College of Medicine, Seoul 03080, \\ Korea; 'Department of Microbiology, Dongguk University College of Medicine, Gyeongju 38066, Korea; ${ }^{3}$ Department of Internal Medicine and Liver \\ Research Institute, Seoul National University Hospital, Seoul National University College of Medicine, Seoul 03080, Korea; ${ }^{4}$ Division of Vectors and \\ Parasitic Diseases, Centers for Disease Control and Prevention, Osong 28159, Korea; ${ }^{5}$ Department of Family Medicine, Seoul National University \\ Hospital, Seoul 03080, Korea; ${ }^{6}$ Department of Family Medicine, Seoul National University College of Medicine, Seoul 03080, Korea; \\ ${ }^{7}$ Division of Allergy and Clinical Immunology, Department of Internal Medicine and Institute of Allergy and Clinical Immunology, \\ Seoul National University College of Medicine, Seoul 03080, Korea;
}

\begin{abstract}
Eosinophilia occurs commonly in many diseases including allergic diseases and helminthic infections. Toxocariasis has been suggested as one cause of eosinophilia. The present study was undertaken to examine the prevalence of toxocariasis in patients with eosinophilia and to identify the risk factors for toxocariasis. This prospective cohort study recruited a total of 81 patients with eosinophilia (34 males and 47 females) who visited the outpatient clinic at Seoul National University Hospital from January 2017 to February 2018 and agreed to participate in this study. The prevalence of toxocariasis was examined by T. canis-specific ELISA, and the various risk factors for toxocariasis were evaluated by a questionnaire survey. Among 81 patients with eosinophilia, 18 were positive for anti-T. canis antibodies (22.2\%); 88.9\% were male (16/18) and 11.1\% were female (2/18). Multivariate statistical analysis revealed that males (OR 21.876, 95\% Cl: 1.667-287.144) with a history of consuming the raw meat or livers of animals (OR 5.899, 95\% Cl: 1.004-34.669) and a heavy alcohol-drinking habit (OR 8.767, 95\% Cl: 1.018-75.497) were at higher risk of toxocariasis in patients with eosinophilia. Toxocariasis should be considered a potential cause of eosinophilia when the patient has a history of eating the raw meat or livers of animals in Korea. A single course of albendazole is recommended to reduce the migration of Toxocara larvae in serologically positive cases with eosinophilia.
\end{abstract}

Key words: Toxocara canis, toxocariasis, eosinophilia, risk factor, raw meat, raw liver

\section{INTRODUCTION}

Eosinophilia is a clinical finding with an increased number of eosinophils in the tissues and/or blood, and it is often recognized based on an elevation of eosinophils in the blood indicated by absolute eosinophil counts more than $500 \mathrm{cells} / \mu \mathrm{l}$ [1]. Eosinophilia occurs in a variety of diseases, including al-

- Received 15 May 2020, revised 30 June 2020, accepted 28 July 2020.

*Corresponding authors (mhchoi@snu.ac.kr; swj0126@amc.seoul.kr)

${ }^{\dagger}$ These authors equally contributed to this work.

"Current address:

Jinwoo Kang: Department of Internal Medicine, SMG-SNU Boramae Medical Center, Seoul 07061, Korea

Woo-Jung Song: Department of Allergy and Clinical Immunology, Asan Medical

Center, University of Ulsan College of Medicine, Seoul 05505, Korea

(c) 2020, Korean Society for Parasitology and Tropical Medicine

This is an Open Access article distributed under the terms of the Creative Commons Attribution Non-Commercial License (https://creativecommons.org/licenses/by-nc/4.0) which permits unrestricted non-commercial use, distribution, and reproduction in any

medium, provided the original work is properly cited. lergic diseases, hypersensitivity reactions to drugs, collagen vascular diseases, helminthic infections, and malignancies $[1,2]$. Helminthiasis is the most common cause of eosinophilia worldwide, while atopic diseases are most commonly associated with eosinophilia in developed countries [2]. Because of the wide range of etiologies, proper diagnosis is necessary for the successful treatment of eosinophilia.

Toxocariasis is one of the most prevalent and widespread parasitic zoonoses in the world, occurring from the sub-Arctic to the tropics [3]. Humans are accidental hosts of Toxocara canis and, less frequently, of Toxocara cati [4] and can be infected by ingestion of contaminated soil, water, or foods where Toxocara eggs or larvae exist [5]. Consumption of raw or undercooked meat or livers from paratenic hosts has been associated with human infections, especially in Asian countries, including Korea and Japan [4,6-8]. Although most infections in humans are asymptomatic, larvae from the eggs or larvae them- 
selves can invade various organs causing clinical syndromes such as visceral larva migrans, ocular toxocariasis, neurotoxocariasis, and covert or common toxocariasis [5].

Eosinophilia is the characteristic clinical sign of helminth infections. In toxocariasis, it occurs as a host immune response against migrating larvae of Toxocara sp. [5] and toxocariasis has been suggested as a common cause of eosinophilia in Korea [7,9-13]. Several papers have reported the possible relationship of eosinophilia with toxocariasis among healthy, asymptomatic adults $[12,14]$ or patients with eosinophilia in Korea $[6,7,9,11,13]$. Some studies were performed by retrospective review of the medical records of eosinophilia patients $[7,9,11]$. This study was performed to investigate the relationship of eosinophilia with toxocariasis through prospective cohort study. Toxocariasis was diagnosed by ELISA using the crude antigen of T. canis larvae, and risk factors for toxocariasis were evaluated by a questionnaire survey.

\section{MATERIALS AND METHODS}

\section{Ethics statement and patient selection}

This study was approved by the Institutional Review Board of Seoul National University Hospital in 2017 (IRB No. H-1612128-818). In this study, inclusion and exclusion criteria were applied to recruit the study subjects. Among the patients who visited the outpatient clinic of the departments of internal medicine and family medicine at Seoul National University Hospital from January 2017 to February 2018, the patients who (1) were older than 18 years old, (2) had blood eosinophil counts exceeding 500 cells/ $\mu l$, and (3) agreed to participate in this study, were considered to have met the inclusion criteria, irrespective of symptom presentation. In contrast, patients who had been diagnosed with asthma, chronic sinusitis, atopic dermatitis, or drug allergy within the year prior were excluded from this study.

\section{Questionnaire survey and clinical characteristics of the subjects}

A structured questionnaire was completed via interview by trained nurses after informed consent was obtained from the subjects. The general information of the enrolled subjects, including sex, age, occupation, and type of residence was obtained. The questionnaire includes a history and a frequency of eating raw meat or viscera (liver or omasum) of cows and other animals, a history of animal contact, and experience with keeping dogs, cats, or other pets. A history of smoking or drinking alcohol was also assessed. The medical history and medication use, current symptoms (if any), and history of taking anthelmintics were acquired. Clinical parameters and imaging studies, if available, were evaluated, and the response to treatment, such as changes in eosinophil counts in the blood, was also analyzed.

\section{ELISA}

The diagnosis of toxocariasis was serologically confirmed by the detection of human IgG antibodies against the crude antigen of T. canis larvae (TCLA) using the in-house ELISA developed by the Institute of Endemic Diseases, Seoul National University College of Medicine [15]. Detection of polyparasitism and consequent cross-reaction with T. canis was also observed by ELISA targeting 4 tissue parasites (Clonorchis sinensis, Paragonimus westermani, metacestodes of Taenia solium, and sparganum) [15].

\section{Statistical analysis}

All data were analyzed with IBM SPSS Statistics ver. 25.0 (SPSS, Chicago, Illinois, USA) and GraphPad Prism 5 (GraphPad, Inc., La Jolla, California, USA). The binary logistic regression model was used to identify variables that might be significantly associated with the outcome variables in the questionnaire survey. Variables with a $P$-value of less than 0.2 in the univariate binary logistic regression analysis were entered into the multivariate binary logistic regression analysis. Odds ratios (ORs) with 95\% confidence intervals (CIs) were estimated to show the strength of the associations, and a $P$-value of less than 0.05 was considered statistically significant. The fitness of the model was checked by using the Hosmer-Lemeshow goodness of fit test, and the significance of the multivariable analysis was 0.771 by the Hosmer-Lemeshow test.

\section{RESULTS}

A total of 81 eosinophilia patients were enrolled in this study. Among them, 34 (42\%) were males, and 47 (58\%) were females. The median age of the patients was 57 years (range, 28-80), and the median eosinophil count was 1,403

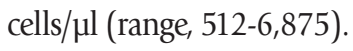

Twenty-eight (34.6\%) of the 81 patients were serologically positive for any parasites by ELISA (Table 1). Among them, 18 patients (22.2\%) were positive for T. canis-specific ELISA, and 
$10(12.3 \%)$ were positive for parasitic antigens other than $T$. canis; 1 for $C$. sinensis, 6 for metacestodes of T. solium, and 3 for both C. sinensis and metacestodes of T. solium (Table 1). Among the patients seropositive for toxocariasis, 14 patients (17.3\%) were T. canis-positive only, and $4(4.9 \%)$ were also weakly positive for other tissue parasites, including C. sinensis, metacestodes of T. solium, and sparganum. Between the T. canis-positive and T. canis-negative groups, the absorbance values of the serum samples were $0.3397 \pm 0.072$ and $0.1070 \pm 0.070$, respectively, which were significantly different between the 2

Table 1. Positive rates of toxocariasis and other parasitic infections in eosinophilia patients by ELISA

\begin{tabular}{lc}
\hline Infection & $\begin{array}{c}\text { Positive } \\
\text { rate (\%) }\end{array}$ \\
\hline No parasite & 65.4 \\
Parasites & 34.6 \\
T. canis only & 17.3 \\
T. canis+C. sinensis & 2.5 \\
T. canis+C. sinensis+metacestodes of $T$. solium & 1.2 \\
T. canis+C. sinensis+metacestodes of $T$. solium+sparganum & 1.2 \\
C. sinensis & 1.2 \\
Metacestodes of $T$. solium & 7.4 \\
C. sinensis+metacestodes of $T$. solium & 3.7 \\
\hline
\end{tabular}

groups $(P<0.001)$.

The final diagnosis in the 53 patients who were serologically negative for any parasite is summarized in Table 2. Among them, idiopathic eosinophilia was the most common (49.1\%), followed by eosinophilic liver abscess (11.3\%), eosinophilic pneumonia (5.7\%) and chronic cholecystitis (5.7\%). Two patients who were serologically negative for $C$. sinensis were final-

Table 2. Final diagnosis on patients serologically negative for any parasites $(n=53)$

\begin{tabular}{lc}
\hline Diagnosis & Frequency (\%) \\
\hline Idiopathic eosinophilia & 49.1 \\
Eosinophilic liver abscess & 11.3 \\
Eosinophilic pneumonia & 5.7 \\
Cholecystitis, chronic & 5.7 \\
Asthma & 3.8 \\
Clonorchiasis & 3.8 \\
Eosinophilic gastroenteritis & 3.8 \\
Pancreatitis, chronic & 3.8 \\
Tuberculosis & 3.8 \\
Allergic rhinitis & 1.9 \\
Cholangitis, acute & 1.9 \\
Drug allergy & 1.9 \\
Hemodialysis related eosinophilia & 1.9 \\
Hypereosinophilic syndrome & 1.9 \\
\hline
\end{tabular}

Table 3. Risk factors of toxocariasis in eosinophilia patients based on the questionnaire survey

\begin{tabular}{|c|c|c|c|c|}
\hline \multirow{2}{*}{ Risk factors } & \multicolumn{2}{|c|}{ Rate (\%) by Toxocara ELISA in } & \multirow{2}{*}{ OR (95\% Cl) } & \multirow{2}{*}{$P$-value } \\
\hline & Positive cases $(n=18)$ & Negative cases $\left(n=53^{a}\right)$ & & \\
\hline \multicolumn{5}{|l|}{ Univariate logistic regression } \\
\hline Male ${ }^{b}$ & $16(88.9)$ & $24(45.3)$ & 9.667 (2.019-46.293) & 0.0045 \\
\hline Age $(y r)$ & $61.11 \pm 9.603$ & $56.60 \pm 13.193$ & $1.031(0.985-1.080)$ & 0.1872 \\
\hline Living with a partner & 11.8 & 15.7 & $0.717(0.137-3.759)$ & 0.6936 \\
\hline Living in a house with a yard & 27.8 & 19.6 & $1.308(0.375-4.561)$ & 0.6739 \\
\hline Preference for raw meat ${ }^{b}$ & 72.2 & 30.8 & $5.850(1.784-19.185)$ & 0.0036 \\
\hline Raw meat & 55.6 & 22.6 & $4.271(1.379-13.228)$ & 0.0118 \\
\hline Raw liver & 33.3 & 11.3 & $3.917(1.071-14.329)$ & 0.0391 \\
\hline Raw omasum & 55.6 & 15.1 & $7.031(2.127-23.246)$ & 0.0014 \\
\hline Eating raw meat $(>1 / \text { month })^{b}$ & 44.4 & 7.7 & $9.600(2.415-38.164)$ & 0.0013 \\
\hline History of contact with animals & 22.2 & 39.2 & $0.443(0.127-1.538)$ & 0.1999 \\
\hline Raising pets & 18.2 & 35.4 & $0.405(0.078-2.094)$ & 0.2810 \\
\hline Currently smoking & 38.5 & 15.2 & $3.482(0.879-13.798)$ & 0.0757 \\
\hline Drinking $>75.6 \mathrm{~g}$ of alcohol per time ${ }^{\mathrm{b}}$ & 33.3 & 12.2 & $2.527(1.091-5.852)$ & 0.0305 \\
\hline Drinking alcohol (>3/week) & 33.3 & 16.7 & $1.657(0.995-2.760)$ & 0.0520 \\
\hline \multicolumn{5}{|l|}{ Multivariate logistic regression } \\
\hline Male ${ }^{b}$ & & & $21.876(1.667-287.144)$ & 0.0188 \\
\hline Preference for raw meat ${ }^{\mathrm{b}}$ & & & $5.899(1.004-34.669)$ & 0.0495 \\
\hline Drinking $>75.6 \mathrm{~g}$ of alcohol per time $\mathrm{b}^{\mathrm{b}}$ & & & $8.767(1.018-75.497)$ & 0.0481 \\
\hline
\end{tabular}

${ }^{a}$ Ten patients who were positive for parasites other than $T$. canis were excluded to prevent the inappropriate confounding of the results from the survey with other possible causes by other parasites.

bStatistically significant. 


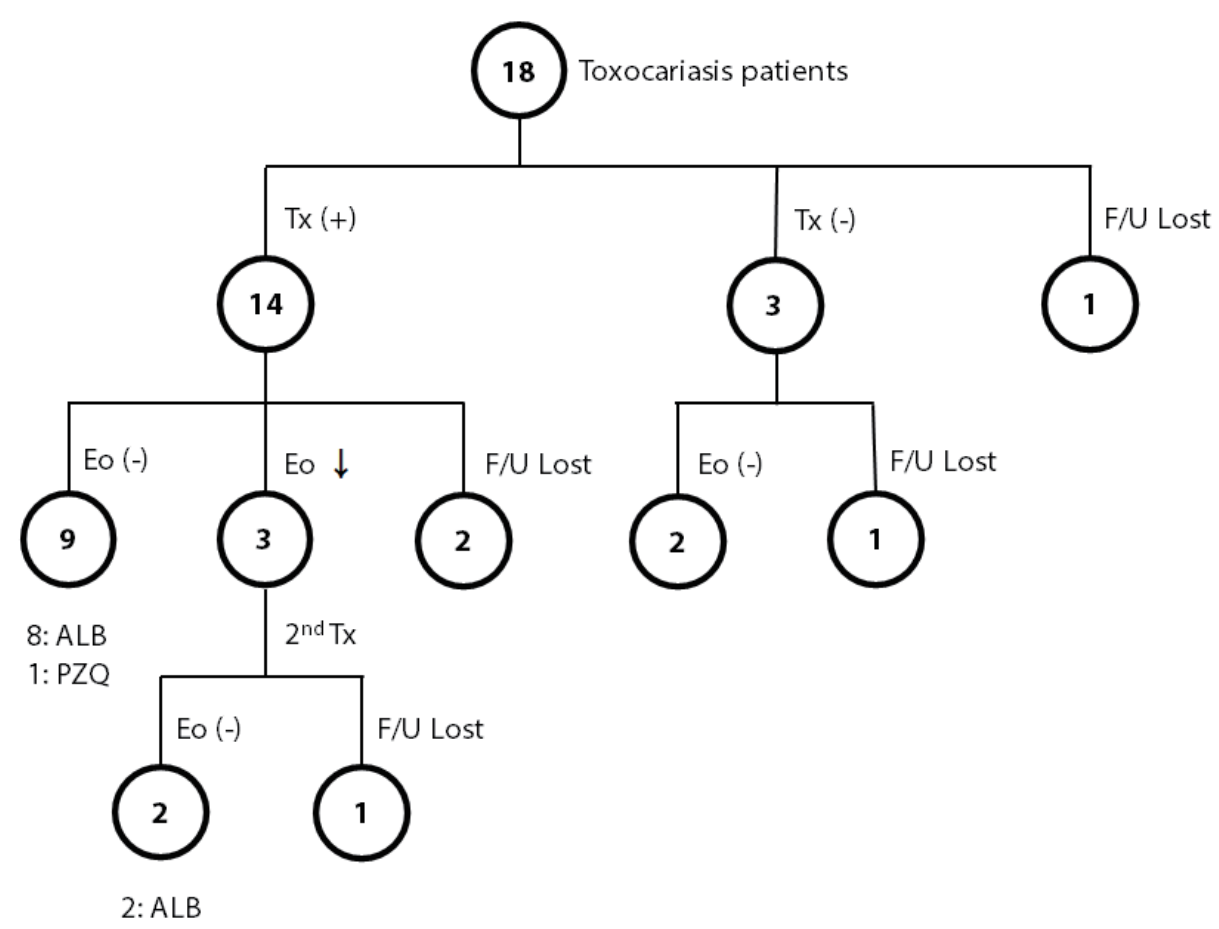

Fig. 1. Summary of the course of eosinophilia in toxocariasis patients with or without anthelmintic treatment. ALB, albendazole; Eo, eosinophilia; F/U, follow-up; PZQ, praziquantel; Tx, treatment.

ly diagnosed with clonorchiasis by stool examination.

The questionnaire survey was performed to identify the possible risk factors that could lead to toxocariasis by comparing the responses of the T. canis-positive and T. canis-negative groups (Table 3). Univariate analysis showed that males were at a significantly higher risk of toxocariasis than females (OR 9.667, 95\% CI: 2.019-46.293). The preference for raw meat, including the livers or omasum of animals (OR 5.850, 95\% CI: 1.784-19.185), history of eating raw meat more than once monthly (OR 9.600, 95\% CI: 2.415-38.164), and history of drinking over $75.6 \mathrm{~g}$ of alcohol per time (OR 2.527, 95\% CI: 1.091-5.852) were also significant risk factors for toxocariasis $(P<0.05)$. However, age, smoking, frequency of drinking, history of contact with animals, raising pets, residence, and the number of family members were not significantly related to toxocariasis. In the multivariate analysis, sex (OR 21.876, 95\% CI: 1.667-287.144), preference for raw meat (OR 5.899, 95\% CI: 1.004-34.669), and a habit of drinking over $75.6 \mathrm{~g}$ of alcohol per time (OR 8.767, 95\% CI: 1.018-75.497) were identified as significant risk factors showing independent correlations with toxocariasis $(P<0.05)$.

The most common symptom (or sign) of toxocariasis patients was an abnormal lab finding (eosinophilia). Most pa- tients showed nonspecific symptoms, including fatigue, dizziness, decreased visual acuity, pruritis abdominal pain, esophageal regurgitation, headache, and cough on their first visit to the outpatient clinic. Among the 18 patients positive for T. $c a-$ nis, 14 patients were treated with anthelmintics, 3 patients were observed without treatment, and one was lost to follow-up (Fig. 1). Among the 14 patients treated, eosinophilia was completely resolved in 9 patients; 8 patients were treated with albendazole, and one was treated with praziquantel. Among the remaining 5 patients in the treated group, 3 patients showed a decreased number of eosinophils, but eosinophilia persisted after treatment, and the other 2 were lost to follow-up. For the 3 patients with persistent eosinophilia after albendazole treatment, 2 were additionally treated with albendazole or praziquantel for each, and both finally achieved normal eosinophil counts. Among 3 of 18 patients who were observed without treatment, all showed a decreased number of eosinophils; 2 became free from eosinophilia, but one still showed persistent eosinophilia during the observation period for up to 1 year.

\section{DISCUSSION}

Parasitic diseases were the most common cause of eosino- 
philia in the present study, and 30 eosinophilia patients (37.0\%) were found to be infected with parasites, including 28 patients diagnosed by ELISA and 2 diagnosed by stool examination. Among the parasitic diseases, toxocariasis was the most common, with positivity in $22.2 \%$ of the patients with eosinophilia. Most patients with toxocariasis were males $(88.9 \%)$, which was consistent with previous reports [7,9$11,13,16]$. The questionnaire survey revealed that dietary habits of eating raw meat or viscera of animals and heavy drinking of alcohol were significant risk factors for toxocariasis as previously reported [6,17].

Toxocariasis can be diagnosed based on the medical history of the patient, clinical findings, and serological tests [18]. The definitive diagnosis is made by direct demonstration of Toxocara sp. larvae in the tissues, but a tissue biopsy is an invasive approach and does not easily identify the larvae due to their small size and extensive distribution $[5,15]$. PCR-based methods targeting the first and second internal transcribed spacers are useful for species identification and genetic analysis of Toxocara spp., but they are not widely used for the diagnosis of toxocariasis [19]. Therefore, ELISA using specific T. canis antigens can be a screening diagnostic method for human toxocariasis.

A commercial ELISA kit using excretory-secretory (ES) antigen derived from second-stage T. canis larvae is available for the serological diagnosis of toxocariasis, with $91 \%$ sensitivity and $86 \%$ specificity [20]. In the present study, in-house ELISA using crude antigen of $T$. canis larvae was used, which showed $92.2 \%$ sensitivity and $86.6 \%$ specificity, similar to those of a commercial ELISA kit [15]. Despite relatively reliable results, ELISA has several limitations: it cannot differentiate between current and past infections and produces cross-reactions with other parasites, including trichinellosis, strongyloidiasis, and fascioliasis $[15,20]$. In the present study, all serum samples were screened with ELISA to rule out the possible cross-reactions with 4 major tissue parasites in Korea.

Previous studies suggested that the geographic location of patients, i.e., whether they were living in urban or rural areas, was a risk factor for toxocariasis because people in rural areas are supposed to be exposed to contaminated soils or contact with dogs more frequently than those in urban areas. Several papers reported a higher prevalence of toxocariasis in rural areas than that in urban areas $[10,14]$. However, another study reported $T$. canis seropositive rate of approximately $5 \%$ among rural adults in Korea [21] and Kim et al. [12] reported a posi- tive rate of $65.0 \%$ for $T$. canis among 97 people with eosinophilia residing in Seoul. Although the information on the locality of residence of the subjects was not collected in the present study, the locality was not a significant risk factor for toxocariasis, as the questionnaire survey showed no significant relation of the history of contact with animals, raising pets, or type of residence with toxocariasis.

The seroprevalence of toxocariasis varied quite widely among the subjects. Several studies have been undertaken to determine the seroprevalence of toxocariasis among healthy people, and most of them reported a high seropositive rate (more than 50\%) for toxocariasis $[10,12,17]$. In regard to patients with eosinophilia, the seropositive rate of toxocariasis ranged from $45.5-86.7 \%[6,7,9,11,13]$. In contrast, the present study revealed a lower positive rate of toxocariasis among eosinophilia patients than previous reports, and Kim et al. [14] reported that $8.7 \%$ of 610 subjects were Toxocara-seropositive among healthy people with eosinophilia. Most studies did not rule out possible cross-reactions, leading to an overestimation of the prevalence of toxocariasis among eosinophilia patients $[6,9,10,13,17]$.

Interestingly, the epidemiological characteristics of toxocariasis are different between Western and Eastern countries. In Western countries, children are more frequently infected with T. canis than adults as they have more chances to come in contact with soil contaminated with T. canis eggs due to geophagia, poor hygiene, or contact with dogs [22]. In contrast, in Eastern countries including Korea and Japan, toxocariasis has affected adults more than children as a food-borne infection that can be acquired by consuming the raw meat or liver of animals infected with larvae of $T$. canis $[6,8,10,11,13]$. Many studies have reported a higher prevalence of toxocariasis among children than adults and demonstrated that the risk factors were a history of contact with infected dogs and of contaminated environments, especially sandboxes $[23,24]$. However, in the present study, contact with animals and raising pets were not risk factors for toxocariasis. Instead, consumption of the raw meat, livers, and omasums of animals was a significant risk factor for toxocariasis, which corresponds with previous reports in Korea $[6,10,11,13,16,17]$.

A variety of animals including mice, rats, cows, pigs, chickens, and lambs, act as paratenic hosts of T. canis $[6,17,22]$ and they are infected through ingestion of embryonated eggs in contaminated soil or by ingestion of larvae encapsulated in the tissues of other infected animals. They can serve as a source 
of infections in humans, especially adults, in countries or ethnic groups in which consumption of raw or undercooked meat or viscera of these paratenic animals is frequent [18]. In Korea, the raw liver of cows is believed to promote good health, especially regarding eyesight, and males enjoy eating raw meat or animal livers more frequently than females [17]. In addition to cow livers, raw livers of pigs, lambs, chickens, and wild-boars are known to be sources of toxocariasis in humans $[18,22,25]$.

In addition to a history of ingesting the raw meat or livers of animals, there have been some debates on the contribution of several behavioral habits, including raising pets, drinking alcohol, and smoking as risk factors for toxocariasis. Choi et al. reported a significant association of raising pets with toxocariasis [17], while other reports showed no relation of raising pets with toxocariasis as in the present study $[6,10]$. According to a previous report, drinking alcohol and smoking were significant risk factors for toxocariasis [14]. However, the present study showed that heavy alcohol consumption was significantly associated with toxocariasis, but the frequency of alcohol consumption was not $(P=0.052)$. Although drinking alcohol and smoking are not direct transmission modes for toxocariasis, they could facilitate the possibility of becoming infected with $T$. canis as people, especially males, have a tendency to eat the raw meat or livers of cows as side dishes while smoking and drinking alcohol in Korea.

The majority of toxocariasis cases are asymptomatic. Therefore, they are often discovered during the investigation of eosinophilia, as in this study, and they are usually self-remitting regardless of treatment. Yoon et al. reported that albendazole treatment and duration of albendazole treatment did not induce a significant difference in the improvement of eosinophilia between the treated and untreated groups of toxocariasis patients [7]. Therefore, they suggested that anthelminthic therapy is not required except for in specific clinical situations, including ocular involvement [7]. However, it is recommended to treat eosinophilia patients when they are diagnosed with toxocariasis, as the larvae can migrate to vital organs, including the eyes and brain, leading to severe sequelae. Albendazole administration at $800 \mathrm{mg} /$ day for 5 days or $10 \mathrm{mg} / \mathrm{kg}$ for 2 weeks is recommended for the treatment of toxocariasis [7].

In the present study, eosinophilia was completely resolved in most toxocariasis patients, regardless of anthelmintic treatment. Eleven of 14 patients treated with anthelmintics and 2 of 3 patients without treatment showed a recovery to the nor- mal level of eosinophils. Eosinophilia due to toxocariasis displayed a higher rate of normalization and resolved more quickly than that due to other causes, with a median time of 3 months irrespective of treatment [7]. Therefore, a 3- or 4-month interval has been suggested as a suitable follow-up period for eosinophilia patients.

In the present study, the absorbance of ELISA for T. canis was not significantly correlated with the eosinophil counts (data not shown), which is in line with a previous report $[12,14]$. Kim et al. reported no significant correlation between the ELISA absorbance and eosinophil counts, but an increase in the antibody-positive rate of T. canis was observed as the eosinophil counts increased [14].

The present study has several limitations. First, this study was performed prospectively for a relatively short period of 13 months, and therefore, the number of enrolled subjects was not large enough to investigate various significant parameters, including the follow-up of the patients after the treatment. Second, the onset of eosinophilia among the subjects was not the same. Therefore, the follow-up period varied depending on the time of inclusion of the subjects and was not long enough to observe the course of remission after treatment. Third, the patients were not recruited evenly across the country and were not categorized by region, urban and rural areas, which might limit the generalization of the findings. Last, children were not included in this study, and therefore, it is difficult to understand the real situation of toxocariasis and possible risk factors in children. These limitations need to be considered in further studies.

In conclusion, the present study demonstrates that toxocariasis is currently one of the most common causes of eosinophilia in Korea. Physicians should consider toxocariasis as a potential cause of eosinophilia, especially in middle-aged males with a recent history of consumption of the raw livers or meat of animals. T. canis-specific ELISA can detect Toxocara infection in clinical eosinophilia patients, and early diagnosis and treatment of toxocariasis can be beneficial for patients by preventing the possible migration of larvae into vital organs during infection. A single course of albendazole is recommended to reduce the migration of Toxocara larvae in serologically positive cases with eosinophilia.

\section{ACKNOWLEDGMENTS}

This research was supported by the Korea Center for Diseas- 
es Control, the Ministry of Health and Welfare (grant number, 2016-265, 114) and, in part, by Seoul National University Hospital. We thank Ms. Hee Jung Lee for performing the T. canis-specific ELISA and the research nurses, Ms. Hye Kyeong Moon and Ms. Su Jung Kim, for their support and assistance in administering the questionnaire survey.

\section{CONFLICT OF INTEREST}

We have no conflict of interest related to this study.

\section{REFERENCES}

1. Lim JH, Lee KS. Eosinophilic infiltration in Korea: idiopathic? Korean J Radiol 2006; 7: 4-6.

2. Rothenberg ME. Eosinophilia. N Engl J Med 1998; 338: 1592-1600.

3. Macpherson $\mathrm{CN}$. The epidemiology and public health importance of toxocariasis: a zoonosis of global importance. Int J Parasitol 2013; 43: 999-1008.

4. Kuenzli E, Neumayr A, Chaney M, Blum J. Toxocariasis-associated cardiac diseases-A systematic review of the literature. Acta Trop 2016; 154: 107-120.

5. Ma G, Holland CV, Wang T, Hofmann A, Fan CK, Maizels RM, Hotez PJ, Gasser RB. Human toxocariasis. Lancet Infect Dis 2018; 18: e14-24.

6. Choi D, Lim JH, Choi DC, Paik SW, Kim SH, Huh S. Toxocariasis and ingestion of raw cow liver in patients with eosinophilia. Korean J Parasitol 2008; 46: 139-143.

7. Yoon SY, Baek S, Park SY, Shin B, Kwon HS, Cho YS, Moon HB, Kim TB. Clinical course and treatment outcomes of toxocariasisrelated eosinophilic disorder. Medicine (Baltimore) 2018; 97: e12361.

8. Yoshikawa M, Nishiofuku M, Moriya K, Ouji Y, Ishizaka S, Kasahara K, Mikasa K, Hirai T, Mizuno Y, Ogawa S, Nakamura T, Maruyama H, Akao N. A familial case of visceral toxocariasis due to consumption of raw bovine liver. Parasitol Int 2008; 57 : 525-529.

9. Kim HB, Seo JW, Lee JH, Choi BS, Park SG. Evaluation of the prevalence and clinical impact of toxocariasis in patients with eosinophilia of unknown origin. Korean J Intern Med 2017; 32: 523-529.

10. Lee JY, Yang MH, Hwang JH, Kang M, Paeng JW, Yune S, Lee BJ, Choi DC. The prevalence of toxocariasis and diagnostic value of serologic tests in asymptomatic Korean adults. Allergy Asthma Immunol Res 2015; 7: 467-475.

11. Kwon NH, Oh MJ, Lee SP, Lee BJ, Choi DC. The prevalence and diagnostic value of toxocariasis in unknown eosinophilia. Ann
Hematol 2006; 85: 233-238.

12. Kim YH, Huh S, Chung YB. Seroprevalence of toxocariasis among healthy people with eosinophilia. Korean J Parasitol 2008; 46: 2932.

13. Seo M, Yoon SC. A seroepidemiological survey of toxocariasis among eosinophilia patients in Chungcheongnam-do. Korean J Parasitol 2012; 50: 249-251.

14. Kim HS, Jin Y, Choi MH, Kim JH, Lee YH, Yoon CH, Hwang EH, Kang H, Ahn SY, Kim GJ, Hong ST. Significance of serum antibody test for toxocariasis in healthy healthcare examinees with eosinophilia in Seoul and Gyeongsangnam-do, Korea. J Korean Med Sci 2014; 29: 1618-1625.

15. Jin Y, Shen C, Huh S, Sohn WM, Choi MH, Hong ST. Serodiagnosis of toxocariasis by ELISA using crude antigen of Toxocara canis larvae. Korean J Parasitol 2013; 51: 433-439.

16. Yoon YS, Lee CH, Kang YA, Kwon SY, Yoon HI, Lee JH, Lee CT. Impact of toxocariasis in patients with unexplained patchy pulmonary infiltrate in Korea. J Korean Med Sci 2009; 24: 40-45.

17. Choi D, Lim JH, Choi DC, Lee KS, Paik SW, Kim SH, Choi YH, Huh S. Transmission of Toxocara canis via ingestion of raw cow liver: a cross-sectional study in healthy adults. Korean J Parasitol 2012; 50: 23-27.

18. Salem G, Schantz P. Toxocaral visceral larva migrans after ingestion of raw lamb liver. Clin Infect Dis 1992; 15: 743-744.

19. Gasser RB. A perfect time to harness advanced molecular technologies to explore the fundamental biology of Toxocara species. Vet Parasitol 2013; 193: 353-364.

20. Jacquier P, Gottstein B, Stingelin Y, Eckert J. Immunodiagnosis of toxocarosis in humans: evaluation of a new enzyme-linked immunosorbent assay kit. J Clin Microbiol 1991; 29: 1831-1835.

21. Park HY, Lee SU, Huh S, Kong Y, Magnaval JF. A seroepidemiological survey for toxocariasis in apparently healthy residents in Gangwon-do, Korea. Korean J Parasitol 2002; 40: 113-117.

22. Fan CK, Lan HS, Hung CC, Chung WC, Liao CW, Du WY, Su KE. Seroepidemiology of Toxocara canis infection among mountain aboriginal adults in Taiwan. Am J Trop Med Hyg 2004; 71: 216-221.

23. Martinez M, Garcia H, Figuera L, Gonzalez V, Lamas F, Lopez K, Mijares V, Corrales Y, Lares M, Ferrer E. Seroprevalence and risk factors of toxocariasis in preschool children in Aragua state, Venezuela. Trans R Soc Trop Med Hyg 2015; 109: 579-588.

24. Sviben M, Cavlek TV, Missoni EM, Galinovic GM. Seroprevalence of Toxocara canis infection among asymptomatic children with eosinophilia in Croatia. J Helminthol 2009; 83: 369-371.

25. Morimatsu Y, Akao N, Akiyoshi H, Kawazu T, Okabe Y, Aizawa H. A familial case of visceral larva migrans after ingestion of raw chicken livers: appearance of specific antibody in bronchoalveolar lavage fluid of the patients. Am J Trop Med Hyg 2006; 75: 303-306. 
